\title{
In vivo OCT imaging of oral cavity tissues
}

\author{
Shu-Fan Chen, Chih-Wei Lu, Yih-Ming Wang, Meng-Tsan Tsai, and C. C. Yang \\ Graduate Institute of Electro-Optical Engineering and Department of Electrical Engineering, \\ National Taiwan University, 1, Roosevelt Road, Section 4, Taipei, Taiwan, R.O.C. \\ (Phone) 886-2-23657624 (Fax) 886-2-23652637 (E-mail) ccy@.cc.ee.ntu.edu.tw \\ Chun-Pin Chiang \\ Department of Dentistry, National Taiwan University, Taipei, Taiwan, R.O.C.
}

\begin{abstract}
We use optical coherence tomography (OCT) with a specially designed probe to structurally image the tissue within the oral cavity. We have imaged different types of healthy oral mucosa, including masticatory mucosa in gingiva and lining mucosa in buccal mucosa. OCT can be used to differentiate between the various types of keratinized and non-keratinized mucosa with high resolution.
\end{abstract}

\section{Introduction}

Because many diseases of oral cavity are accompanied by the changes of tissue structures, high-resolution diagnostic imaging is therefore crucial to the diagnosis and treatment of these diseases. Conventional X-ray radiography can provide quite high resolution, suitable for characterizing macroscopic structural changes in teeth. Ultrasound is limited by the wavelength of the source. Sub-millimeter clinical resolutions of ultrasound can be achieved only under the optimal conditions. The increased image acquisition times necessary to achieve sub-millimeter resolution in MRI, as well as its physical footprint and expense, make it impractical in routine clinical dentistry settings.

The development of optical coherence tomography (OCT) has made it possible to overcome these limitations by using interferometric cross-correlation techniques to detect the coherent backscattered components of short coherence-length light [1-3]. Pioneered by Fujimoto and co-workers to perform in vivo clinical imaging of the human eye [4], OCT has been an emerging bio-imaging technology that promises to have broad and significant impact on clinical diagnostic imaging.

In this paper, we present an investigation of OCT in vivo imaging of tissue structures in the oral cavity. We have performed imaging of several types of oral mucosa. The results indicate that OCT is capable of high resolution imaging in a substantial number of clinical dental applications.

\section{Experimental Setup}

To perform OCT imaging of oral mucosa, we used a compact, fiber-based OCT scanner. The super-luminescent diode operating at $950 \mathrm{~nm}(\Delta \lambda=65 \mathrm{~nm})$ served as the light source, producing $1.5 \mathrm{~mW}$ power. The operation of the OCT scanner was completely automated and controlled by a personal computer. A transverse scan of $1 \mathrm{~mm}$ in length could be completed within 2 seconds. Depth scanning (penetration into tissues) was achieved with a phase delay line in the reference arm. The in-depth resolution of the OCT system is around $10 \mu \mathrm{m}$ in free space. To facilitate the access to the tissue in the oral cavity, we constructed an L-shaped probe with a linear motor. The use of this probe for scanning any area of oral mucosa is quite convenient. The picture of the probe is shown in Fig. 1.

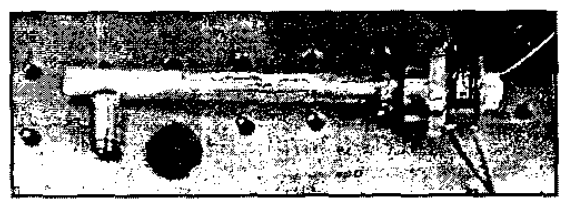

Fig. 1 The scanning probe in the sample arm. The lenoth is around in $\mathrm{cm}$ 


\section{Experimental Results}

Oral mucosa can be divided into three types: the masticatory mucosa (gingival and hard palate mucosa), the lining mucosa (alveolar, soft palate, labial, and buccal mucosa, as well as the mucosa of the mouth floor and the ventral surface of the tongue), and the specialized mucosa (lips, dorsum of the tongue). The scanning image of one type of masticatory mucosa (gingival mucosa) is shown in Fig. 2. The submucosa is not clear here. The $170-\mu \mathrm{m}$ thick layer at the top of the tissue is referred to as epithelium (EP), including the keratin layer. The $280-\mu \mathrm{m}$-thick region beneath the epithelium is the lamina propria (LP). The layer beneath lamina propria is submucosa. The total depth of OCT imaging in the gingival mucosa is about $700 \mu \mathrm{m}$.

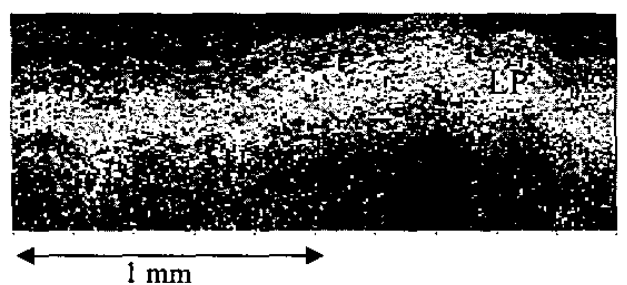

Fig. 2 OCT image of gingival mucosa. The epithelium (EP) appears as the $170 \mu \mathrm{m}$ top layer above the $280 \mu \mathrm{m}$ thick lamina propria (LP).

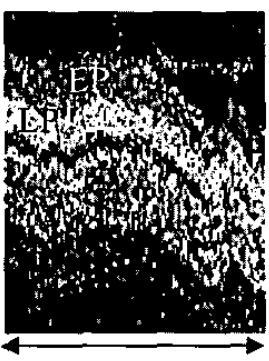

$1 \mathrm{~mm}$

Fig. 3 OCT image of buccal mucosa. The epithelium (EP) appears as the $220 \mu \mathrm{m}$ top layer.

The scanning image of another type of lining mucosa (buccal mucosa) is shown in Fig. 3. Lining mucosa differs structurally from the masticatory one. Besides, we can see the submucosa and connective tissue clearly. The thickness of epithelium (EP) is around $220 \mu \mathrm{m}$, and lamina propria (LP) is $240 \mu \mathrm{m}$. The LP smoothly penetrates into the submucosa, which is well developed $500 \mu \mathrm{m}$ deep. The total depth of OCT imaging in the buccal mucosa is about $900 \mu \mathrm{m}$.

\section{Conclusion}

In vivo imaging of the human oral cavity has been demonstrated using a fiber-based OCT system. We have imaged several types of oral mucosa and have proved that OCT is a good diagnostic tool in dental clinics. This OCT system will be used for early diagnosis of oral cancer.

\section{References}

[1] D. Huang, E. A. Swanson, C. P. Lin, J. S. Schuman, W. G. Stinson, W. Chang, M. R. Hee, T. Flotte, K. Gregory, C. A. Puliafito, and J. G. Fujimoto, "Optical Coherence Tomography," Science, 254, 1178 (1991).

[2] M. R. Lee, J. A. Izatt, E. A. Swanson, D. Huang, J. S. Schumun, C. P. Lin, C. A. Puliafito, and J. G. Fujimoto, IEEE Eng. Med. Biol. Mag. 14, 67 (1995).

[3] J. G. Fujimoto, M. E. Brezinski, G. T. Tearney, S. A. Boppart, B. E. Bouma, M. R. Hee, J. F. Southern, and E. A. Swanson, "Biomedical imaging and optical biopsy using optical coherence tomography," Nature Medicine, 1, 970-972 (1995).

[4] C. A. Puliafito, M. R. Hee, J. S. Schuman, and J. G. Fujimoto, "Optical Coherence Tomography of Ocular Diseases", SLACK, Thorofare, NJ, (1996). 